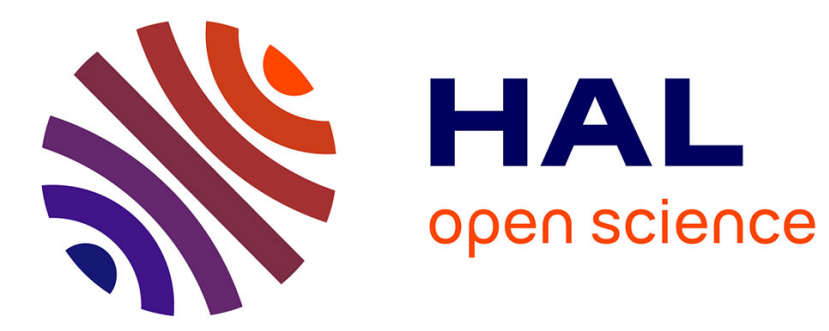

\title{
An experimental investigation of imprecision attitude and its relation with risk attitude and impatience
}

\author{
Michèle Cohen, Jean-Marc Tallon, Jean-Christophe Vergnaud
}

\section{To cite this version:}

Michèle Cohen, Jean-Marc Tallon, Jean-Christophe Vergnaud. An experimental investigation of imprecision attitude and its relation with risk attitude and impatience. Theory and Decision, 2011, 71 (1), pp.81-110. 10.1007/s11238-010-9205-8 . halshs-00502820

\section{HAL Id: halshs-00502820 \\ https://shs.hal.science/halshs-00502820}

Submitted on 20 Jul 2010

HAL is a multi-disciplinary open access archive for the deposit and dissemination of scientific research documents, whether they are published or not. The documents may come from teaching and research institutions in France or abroad, or from public or private research centers.
L'archive ouverte pluridisciplinaire HAL, est destinée au dépôt et à la diffusion de documents scientifiques de niveau recherche, publiés ou non, émanant des établissements d'enseignement et de recherche français ou étrangers, des laboratoires publics ou privés. 


\title{
An experimental investigation of imprecision attitude and its relation with risk attitude and impatience*
}

\author{
Michèle Cohen† Jean-Marc Tallon; Jean-Christophe Vergnaud $\$ ₫$
}

July 2009

\begin{abstract}
We report in this paper the result of three experiments on risk, ambiguity and time attitude. The first two differed by the population considered (students vs. general population) while the third one used a different protocol and concerned students and portfolio managers. We find quite a lot of heterogeneity at the individual level. Of principal interest was the elicitation of risk, time and ambiguity attitudes and the relationship among these (model free) measures. We find that on the student population, there is essentially no correlation. A non negligible fraction of the population behaves in an extremely cautious manner in the risk and ambiguity domain. When we drop this population from the sample, the correlation between our measures is also non significant. We also raise three questions linked to measurement of ambiguity attitudes that come out from our data sets.
\end{abstract}

Keywords: Experiments, Risk aversion, Impatience, Imprecision Aversion.

JEL Classification number: C90, D81, C91

\section{Introduction}

We report in this paper the results of an experimental investigation in three domains, i.e., risk, ambiguity and time attitude. While risk and time attitude have been extensively studied, there has been fewer attempts in the economics literature to quantify ambiguity attitude and to relate it to risk attitude in particular.

Ellsberg examples convincingly show that individual's choices in a context of ambiguity (i.e., partial information on the odds of winning) cannot be explained by the subjective expected utility model, widely adopted in economics, even with high degrees of risk aversion. Subjects react to the lack of information not by putting subjective probabilities on "ambiguous" events,

\footnotetext{
${ }^{*}$ We thank for useful discussions and comments Luc Arrondel, Syngjoo Choi, Glenn Harrison, Jean-Yves Jaffray, Graham Loomes, André Masson, Bernard Salanié, Karine Van der Straeten, Peter Wakker, Martin Weber, participants at FUR XIII in Barcelona, D-TEA at HEC, RUD 2009 at Duke University, and seminar audience at Toulouse School of Economics and Westminster Business School. We thank Maxim Frolov, Nicolas Roux for their help with the experiments and valuable research assistance. We gratefully acknowledge financial support from ANR Grant Princes ANR-05-BLAN-0270 as well as ANR Grant Risk Attitude ANR-05-BLAN-0345. Finally, we thank Véronique Noiville, from TNS-Sofres for her help in developing part of this project.

$\dagger$ Paris School of Economics, U. Paris I Panthéon-Sorbonne. michelecohen@univ-paris1.fr

${ }^{\ddagger}$ Paris School of Economics, U. Paris I Panthéon-Sorbonne, CNRS. jean-marc.tallon@univ-paris1.fr

$\S$ Centre d'économie de la Sorbonne, CNRS. jean-christophe.vergnaud@univ-paris1.fr

『Address (all authors): Centre d'économie de la Sorbonne, 106 bd de l'Hôpital, 75013 Paris, France.
} 
but rather by preferring acts that are not prone to such ambiguity. To the best of our knowledge, most experimental studies ${ }^{1}$ on ambiguity aversion consider only simple and extreme cases, e.g., no information vs precise (and equiprobable) information in the case of Ellsberg two urn. In this paper, we expose a simple experimental protocol aimed at eliciting people's attitude toward ambiguity (or imprecision as we shall call it from now on when we talk about our experiment), in a model-free way. The protocol is based on the same idea as Ellsberg two-urn example, in which we allow for a wider range of information configuration. By changing the information available to the decision maker, it allows to clearly separate information imprecision from aversion to imprecise information. This distinction is not made in traditional Ellsberg experiments since information is kept fixed.

Although this design is inspired by recent theoretical work ${ }^{2}$ that do provide preference representation theorems with a clear separation of (objective) information imprecision from (subjective) aversion to it, our design is not meant to test any particular model of decision making under ambiguity developed recently. ${ }^{3}$ It rather aims at providing as much as possible model free measures of various attitudes and assess their determinants as well as their relationships to one another. In particular, once imprecision attitudes are elicited together with usual measures of risk aversion and impatience, we can study the correlations between these three measures (with special attention to the correlation between risk and imprecision attitudes.) Is imprecision aversion simply a feature akin to extreme risk aversion or does it represent a behavioral feature independent of traditional risk aversion? We view the answer to this question as important from a theoretical point of view: if measures are correlated, we should develop axiomatic models taking this into account, or at least explore further what this correlation means in terms of simple behavioral axioms; it the measure are not correlated, then this gives support to existing models of ambiguity aversion in which risk and ambiguity attitudes are captured independently. The answer is also important from an empirical point of view: if for instance risk and imprecision measures are not correlated and we have reasons to think that both are relevant to explain say financial decisions, such measures should be included in surveys or any investigations of financial behavior. Another important question that we can address with our data is whether the general population is different or not from the student population generally studied in experiments, from the perspective of risk, ambiguity and time attitudes.

The data comes from three experiments. The first two were part of a larger project and differ mostly by the population considered (students vs. general population). The third one was more focussed on risk and imprecision aversion and was run both on students and on 16 portfolio managers. When looking at each attitude separately, we find relatively consistent behavior

\footnotetext{
${ }^{1}$ See below the discussion of related literature.

${ }^{2}$ In particular Gajdos, Hayashi, Tallon, and Vergnaud (2008) as developed in Section 6.

${ }^{3}$ For instance, Choquet expected utility of Schmeidler (1989), multiple prior of Gilboa and Schmeidler (1989) and its extension to $\alpha$-MMEU, variational preferences of Maccheroni, Marinacci, and Rustichini (2005), smooth ambiguity of Klibanoff, Marinacci, and Mukerji (2005) or the contraction model of Gajdos, Hayashi, Tallon, and Vergnaud (2008).
} 
within each domain (measured by correlation between answers in the various questions asked in each domain) in all three experiments. Overall, aversion to imprecision is significant in all three experiments while risk aversion is not that important. We uncover, in the general population, a significant fraction of subjects that behave in the risk and the imprecision domains in a rather extreme manner: when asked to choose between a lottery or a sure amount they always opt for the latter and similarly when asked to choose between an imprecise lottery and a precise lottery they always choose the latter. This reflects extremely (unreasonably?) cautious behavior. Still in the general population we can look into the determinants of various attitudes. Usual sociodemographic variables are not significant to predict behavior in the population composed of nonextreme subjects. On the other hand, the chance to behave in an extremely cautious manner is positively affected by age, and negatively affected by education. We also find a gender difference concerning time attitude, but only in terms of choices of a present reward vs. a future reward and not when looking at rewards in the future vs. rewards in an even more distant future.

When assessing the relationship among our three experimental measures of, respectively, risk, ambiguity, and time attitudes, we find that in the student population there is virtually no correlation among them. On the general population, the correlation among risk and imprecision aversion is equal to .22 . However, this correlation is completely driven by the existence of extreme subjects. Once removed from the sample, the correlation is equal to zero.

Why might our results be of interest? Assessing the inter-relationship among the three feature seems interesting per se, since theory is silent about any such relation. A general conclusion is thus that we do need these three measures as one cannot be deduced from the other. If we had found say perfect correlation, this would have meant that explaining a given behavior by e.g. impatience has not much sense since it could be also explained (modulo various non-linearities in the way the parameters enter the functional forms) by risk aversion. This also gives hope to identify in a precise manner which attitude plays a crucial role in explaining various phenomena. Indeed, eventually, we want to use the measures exposed here to say something about "real behavior" (outside the lab). If parameters are related to one another, it might restrict the scope for explaining financial puzzles such as, say, the equity premium. To explain part of this puzzle by ambiguity aversion, it is important to be able to vary risk and ambiguity aversion separately. ${ }^{4}$

Our study also reveals some discrepancies among various ways of approaching the measurement of risk, ambiguity, and time attitudes. In the general population experiment, subjects were also asked to report on a scale how risk averse and impatient they were. Self reported assessments of attitude toward risk/uncertainty give a somewhat different picture as the one based on experimental data. Similarly, impatience scales are weakly correlated with experimental measure of impatience. Second, we asked subjects, in a separate questionnaire, to answer to hypothetical lotteries and thus can assess the link between this measure of risk aversion and the (incentivized) experimental measure. Finally, in the third experiment we also asked the (symmetrized version of the) three color Ellsberg urn. Our measure of imprecision aversion (based on Ellsberg two

\footnotetext{
${ }^{4}$ See Ju and Miao (2008) and Collard, Mukerji, Sheppard, and Tallon (2009).
} 
urn like questions) was not instrumental to predict accurately the choice in the three color urn, although a more qualitative measure based on the experiment is indeed significant. These findings call for more research on the precise link between these various ways of assessing a priori similar behavioral features and how these measures are affected by framing issues.

\section{Related literature}

There is evidently a large literature on experiments on risk preferences and time preferences. There are fewer studies (although their number is rising rapidly) aimed at quantifying ambiguity or imprecision aversion and even fewer that relate measures on these three dimensions.

Cohen, Jaffray, and Said (1987) is one of the first paper to experimentally find an absence of correlation between risk and ambiguity attitudes (in a context of "complete ignorance", that is with no information at all on the content of the urns.) See also Di Mauro and Maffioletti (2004). Lauriola and Levin (2001) report the results of an experiment in which they ask subjects to choose between a completely unknown urn and a known urn, with the odds of winning changing from one question to another. They find a positive correlation between risk and imprecision attitudes in the loss domain but none in the gain domain. They also show that the existence of a correlation is due to "extreme" subjects in their sample. Chakravarty and Roy (2009) find a positive correlation between risk and ambiguity aversion. Their protocol does not entail any change in imprecise information. All these studies were done on a population of students.

There has been a recent surge in research to estimate various preference parameters on a larger population, via experimental or survey data. ${ }^{5}$ van Praag and Booij (forthcoming) show a moderate negative correlation between risk aversion and time discount: "prudent" people take fewer risks and plan things ahead. They furthermore show that for big prizes, risk aversion cannot be estimated correctly without taking into account the time dimension (a large prize will likely be consumed over a time horizon of several years). Andersen, Harrison, Lau, and Rutström (2008) elicit jointly risk and time preferences using field data and show that this results in significantly lower discount rates than separate elicitation of discount rates. Tanaka, Camerer, and Nguyen (2008) study how risk and time preferences are linked with wealth but do not assess the correlation between these two preference dimensions. This paper shares with von Gaudecker, van Soest, and Wengstrom (2009) the idea that risk (and time) attitude has many dimension to it, including loss aversion or timing of resolution of uncertainty. We abstract from this since our experiment did not entail loss or multi-stage lotteries. Dohmen, Falk, Huffman, Sunde, Schupp, and Wagner (2005) report results from a large scale survey that had general risk question similar to our survey. They also had an experimental part for a sub sample. They find that a binary variable constructed from answers to the risk question in the survey is predictive of the subjects' actual choice in the lottery experiment. These studies did not deal with ambiguity/imprecision

\footnotetext{
${ }^{5}$ See e.g., Tanaka, Camerer, and Nguyen (2008), von Gaudecker, van Soest, and Wengstrom (2009), Dohmen, Falk, Huffman, Sunde, Schupp, and Wagner (2005), Harrison, Lau, and Rutström (2007), Harrison, Lau, and Williams (2002), Guiso and Paiella (2008), van Praag and Booij (forthcoming), Guiso and Jappelli (2008), Burks, Carpenter, Gotte, and Rustichini (2008), Dohmen, Falk, Huffman, and Sunde (2008)...
} 
aversion. Guiso and Jappelli (2008) conducted a survey on some Italian bank's clients. They find a positive correlation between answers to questions about risk and imprecision attitude. They relate this to modes of decision making (intuitive vs. reasoned). Cabantous (2007) surveyed insurance professionals and found that imprecision aversion was pervasive in this population. She also finds that sources of ambiguity (conflict of expert opinion or imprecision) matter. She did not assess risk aversion simultaneously. Burks, Carpenter, Gotte, and Rustichini (2008) use data collected among truck drivers and show that there is a positive and strong correlation between risk and ambiguity aversion. They show that a common factor, cognitive ability, explain many features of these subjects. Dohmen, Falk, Huffman, and Sunde (2008) also find that lower cognitive ability is associated with greater risk aversion and more pronounced impatience.

The closest experimental design aimed at eliciting imprecision aversion is the one of Hayashi and Wada (forthcoming). ${ }^{6}$ Their aim was different though since they wanted to compare the various theoretical models mentioned above. By giving information to subjects in the form of sets of probability distribution (over three states), they find that subjects are sensitive to the dimension and the shape of the sets and not only to the worst- and best-case, thus violating the $\alpha$-MMEU models. They also show that the presence of non-extreme points matter, in contradiction to what the contraction model assumes. They find mixed support for the secondorder prior model. Andersen, Fountain, Harrison, and Rutström (2009) estimate a second-order prior model in which they jointly elicit risk and ambiguity attitude as well as subjective beliefs. They show that ambiguity aversion is quantitatively significant. They also show that attitude towards risk and uncertainty can be different, quantitatively and qualitatively. Ahn, Choi, Gale, and Kariv (2009) also reports an experimental design that allows them to quantify imprecision aversion in various models. Their task is cast in a portfolio choice problem. They did not change the information available to subjects but rather the returns of the asset in the various states. Contrary to Hayashi and Wada (forthcoming) their results tend to show that most subjects' behavior is better explained by the $\alpha$-MMEU or the contraction model than by the second-order prior model. They find quite a lot of heterogeneity at the individual level. Hey, Lotito, and Maffioletti (2008) propose a different way of implementing ambiguity in the lab and find that most theories perform rather poorly. Finally, Potamites and Zhang (2007) present a field experiment on ambiguity aversion among investors in China. They report, on this peculiar population, a small positive correlation (.085) between risk aversion and ambiguity aversion not significantly different from zero.

\footnotetext{
${ }^{6}$ A somewhat older literature also tried to quantify ambiguity aversion, either through the elicitation of willingness to pay for ambiguous prospects or by comparing simple information structures. See e.g. Yates and Zukowski (1976), Curley and Yates (1985), Fox and Tversky (1995) and Chow and Sarin (2002). The last two papers question the realm of ambiguity aversion in that they find very different willingness to pay for an ambiguous prospect, depending on whether an unambiguous prospect was also evaluated or not.
} 


\section{Outline of the paper}

As mentioned, the results we report are based on three experiments. The first two were very similar in their design but differed regarding the population (students versus general population). The third one was ran on students and portfolio managers and its design was slightly different. In the text we will first focus on the general population experiment (experiment 2) that was run via the web. We present most of our results in this case giving only a partial account of the data of this experiment in the student population, when it yields results that differ from those in the general population. We then confirm the absence of correlation among various attitudes based on the third experiment, which was more focussed and thus provided more data (in a different format) for each subject.

In Section 2 we give a simple description of the protocol for the general population. In Section 3 we present our measures of the different attitudes and assess the consistency of the subjects' choices. Determinants of these attitudes are briefly described in Section 4. Section 5 reports the correlations among the three attitudes. In Section 6 we concentrate on the more focussed protocol and provide further evidence of absence of correlation among the three attitudes. Section 7 relates three puzzles in the subjects' choices. Section 8 concludes. An Appendix contains more details on the various protocols implemented.

\section{Experimental design}

We give here an overview of the online experiment ("experiment 2") we ran on a sample of the French population. Details are in the Appendix. The web experiment was run on a sample of 400 people from the general French population. In this study a survey questionnaire, asking information about behavior in various domains (health, consumption, leisure, finance...) as well as background information, was sent, through the poll institute TNS-Sofres to a sample of 4000 persons. A sub sample of 400 people subsequently participated to the on line experiment, which was about 20 minutes long. Subjects were paid in gift certificates by TNS-Sofres.

Subjects had to answer questions in the risk domain, the imprecision domain and the time domain. The three risk questions correspond to three different lotteries with winning probability $.5, .3$, and .7 and gains 20,30 , and $15 €$ respectively. We asked a series of choice between these lotteries and certain monetary amounts, along the bracketing technique.

The general idea behind the imprecision questions is to generalize Ellsberg's famous two-urn examples by varying the information available. The imprecision questions were simple choices between a given lottery with known odds and an imprecise lottery, in which the chance of winning was said to be between bounds $a$ and $b .^{7}$ A typical series of question was as follows: do you prefer a gain of 20 euros contingent on drawing a winning ball from a 10 ball urn that contains between $a$ and $b$ winning balls or a gain of 20 euros contingent on drawing a winning ball from a 10 ball urn that contains exactly 5 winning balls. We elaborated an original payment scheme

\footnotetext{
${ }^{7}$ See Table 12 in the Appendix for an explicit description of this task.
} 
(detailed in the Appendix) aimed at implementing "true" imprecision, without having to appeal to second order probability distributions. ${ }^{8}$

In the time domain, given that we could not implement immediate payment (contrary to the experiment ran on students), we asked only two questions in which the subjects had to choose between a 100 euros payment in a month and $100+x$ euros in two months (resp. seven months). In the first series, $x$ went from 0 to 20 , while it went from 0 to 50 in the second series (seven months horizon.)

\section{Measures of different attitudes}

The different parts of the protocol all have in common a basic structure, that is, making binary choices: between lotteries and certain amounts, imprecise lotteries and lotteries, money earlier and money later. Thus, we take as a measure of risk aversion for a subject the number of times he chose a certain amount rather than the lottery: for an agent whose choices are monotonic, the higher this number, the higher the subject's risk aversion. Call this variable NCertain.

In a similar vein, we use the number of times a subject chose the precise lottery over the imprecise lottery to assess his imprecision aversion. The higher this number, the more imprecision averse the subject. Again, this provides the same information as the estimation of a "probabilistic equivalent" for that imprecise lottery if the subject is consistent. Call this variable NPrecise. For monotonic agents, this number is increasing with imprecision aversion. For time questions, we count the number of times the subject chose the more distant option. The higher the number, the more patient is the subject. Call this variable NDelayed. For monotonic agents, this number is increasing with patience. To sum up, the count measure has the advantage of using all the data but the drawback to potentially treat in the same way two very different individuals (if one is irrational). In the data, we however do not see a lot of inconsistencies, but rather occasional mistakes (a lot of the subjects who made a mistake in the risk or imprecision questions did so for the first question and did not violate monotonicity afterward) so that the count measures are fairly accurate.

Non monotonicity is a problem in the data only for the risk and imprecision questions, which were of the "bracketing type". For time questions, that were asked in a monotonic way (from the smallest amount to the highest amount in the farther future) we did not expect much (and indeed did not observe much) non-monotonicity. Given the nature of the online experiment, we expected to see a fairly high percentage of violations of monotonicity since instructions were not carefully read by an instructor but simply read (or not) on the screen by the participants and we could not monitor where the participants filled out the questionnaire (at home, at work, internet cafe etc). Out of 400 participants, $46.5 \%$ (186 subjects) did not violate monotonicity in any of the 6 risk and imprecision questions, $31.25 \%$ violated monotonicity only once and $14.5 \%$ twice.

\footnotetext{
${ }^{8}$ The payment scheme for the online experiment was different than the one implemented for students, as explained in the Appendix. Both schemes are, to the best of our knowledge, new in the literature -although the design of Hayashi and Wada (forthcoming) is similar in spirit to the one implemented for the web experiment.
} 
Most of our results (in particular the absence of correlation) are not affected when we deal with the sub sample of monotonic subjects. Behaving in a monotonic way in all the questions is not explained by characteristics such as age, sex, income and education.

\subsection{Risk attitude}

We find overall rather small mean risk aversion, together with quite a lot of heterogeneity. Lottery questions were such that a risk neutral subject would have NCertain equal to 16 or 17 (depending on how indifference is broken). We find that the mean answer is 19.76 ( $\mathrm{std}=8.3$ -median=18.5). Risk aversion in the "general population" is both higher on average and more dispersed than in the student population. This comes from a particular feature observed in this experiment: quite a lot of subjects (47) always chose the safe amount rather than the lottery. These subjects exhibit a very high degree of risk aversion as they prefer for instance a sure 2 euros gain rather than playing a lottery with $50 \%$ chance of winning 20 euros.

If we drop these 47 subjects (the ones that constitute the peak on figure 1) and consider the 353 remaining subjects, the mean and standard deviations drop (mean=17.86, std: 6.86 - median $=18$ ) to a level close to what was observed on the student population.

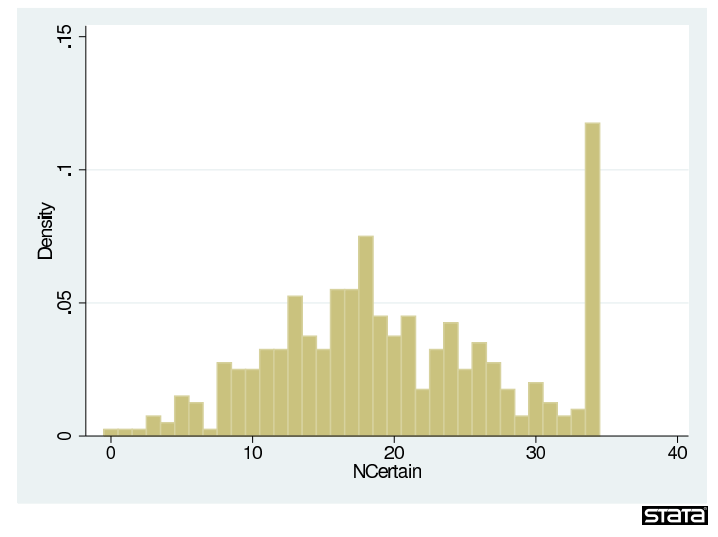

Figure 1: Histogram of NCertain, Web experiment (TNS-Sofres, 400 subjects).

\subsection{Imprecision attitude}

The main finding we relate in this section is that we observe quite a lot of aversion to imprecision. In our online experiment, imprecision neutral subjects ${ }^{9}$ would have answered from 5 to 8 times (because of possible indifference) the precise lottery over the imprecise lottery. The average number of choices of the precise lottery is 14.3 ( $\mathrm{std}=4.1$-median=15). For the 186 monotone subjects, the average is $15.4(\mathrm{std}=4.5-$ median $=17)$. The average drops to 13.27 when we drop the 69 subjects that always chose the precise option. The general population exhibits higher imprecision aversion than the student population. But again, as in the risk questions, the

\footnotetext{
${ }^{9}$ Recall that imprecision neutral subjects evaluate an imprecise lottery by its median probability of winning.
} 
difference is mainly due to the presence of extremely imprecision averse decision makers, as captured by the peak at the upper end of the histogram. These are subjects who, no matter what the odds were, always preferred the risky option over the imprecise option.

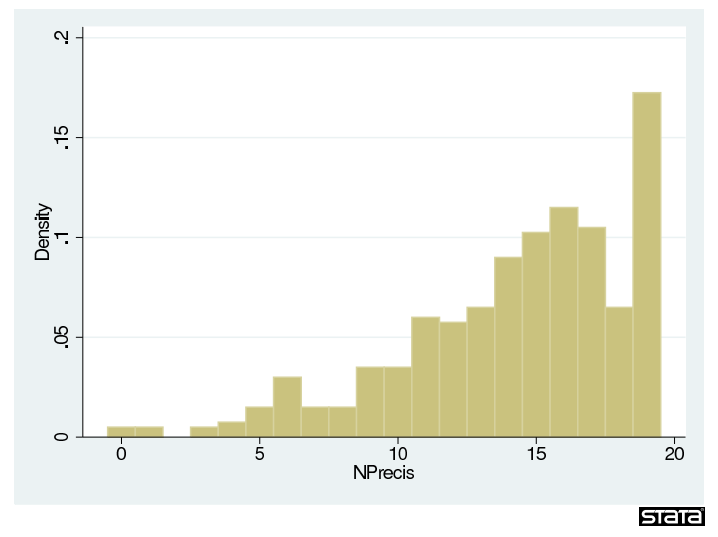

Figure 2: Histogram of NPrecise, Web experiment (TNS-Sofres, 400 subjects).

We can thus conclude that, in both the risk and the imprecision domains, the student and the general population differ only to the extent that, in the latter, there exists a non negligible fraction of subjects, 35 out of 400 , who answered in an extremely cautious way.

\subsection{Time attitude}

We report here the graph of the number of delayed answers in the online experiment. The mean answer is $9.5(\mathrm{std}=4.9-$ median $=10)$. The two other experiments were identical to the one online except that there was a first question involving a choice between money today and money in a month. Recall that the first was ran on students while the third one was ran both on students and portfolio managers.

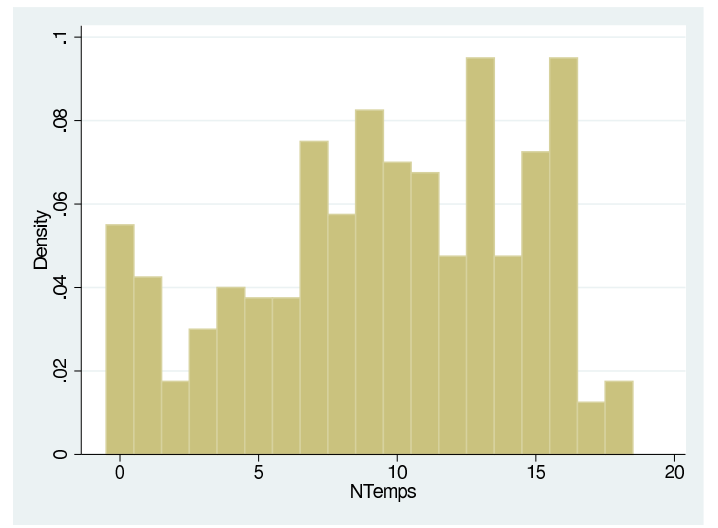

Figure 3: Histogram of NDelayed, Web experiment (TNS-Sofres, 400 subjects).

We did not find much difference between the student population in experiment 1 and 3 (although the time questions in experiment 3 were not payed.) However, professionals appeared 
more patient than students. Table 1 reports the decomposition of the overall measure question by question. We see that the student and general population is rather homogeneous in its answers while the professionals are "uniformly" more patient. The difference between professionals and the student population answers is decreasing with the time horizon: it is larger in the first two questions (where the horizon is 1 month) than in the third (horizon of 6 months). We also find that the difference is slightly higher in the first question than in the second, pointing to a possible interpretation of greater "impulsiveness" (on top of higher impatience) for students compared to professionals. The effect however is rather small.

\begin{tabular}{cccc} 
NDelayed & Now vs 1 month & 1 vs 2 months & 1 vs 7 months \\
\hline \hline Expe. 1 & 4.7 & 4.13 & 6.3 \\
Expe. 2 & $(3.5)$ & $(3.6)$ & $(3.6)$ \\
& $\mathrm{xx}$ & 4.45 & 5.1 \\
Expe. 3-students & $\mathrm{xx}$ & $(2.5)$ & $(3.0)$ \\
& 4.4 & 3.8 & 5.7 \\
Expe. 3-professionals & $(3.5)$ & $(3.3)$ & $(3.3)$ \\
& 7.4 & 6.7 & 7.06 \\
& $(2.8)$ & $(3.3)$ & $(2.5)$ \\
\hline
\end{tabular}

Table 1: Mean number of delayed answers for the three experiments. In parenthesis, standard deviation.

\subsection{Consistency within each domain}

In this section, we look at the consistency of the subjects within each domain. We use a simple measure, namely the correlation among the questions pertaining to a particular attitude. Overall, there is a pretty high correlation among the various questions in each domain pointing to a large degree of consistency of the subjects.

\begin{tabular}{|c|c|c|}
\hline & Lottery 2 & Lottery 3 \\
\hline Lottery 1 & $.8^{\star \star \star}\left(.76^{\star \star \star}\right)$ & $.69^{\star \star \star}\left(.6^{\star \star \star}\right)$ \\
\hline Lottery 2 & 1 & $.69^{\star \star \star}\left(.6^{\star \star \star}\right)$ \\
\hline
\end{tabular}

Table 2: Experiment 2 (web). Correlation among the three risk questions. 400 subjects. In parenthesis, correlation without the extreme subjects (365 subjects).

We find high and highly significant correlations within each domain. The correlation is slightly smaller when we drop the 35 "extreme" subjects, that always - and consistently-chose the sure outcome in the three risk questions and the precise lottery in the three imprecision questions. Correlation is higher in the risk domain than in the imprecision domain, both being significant at the $1 \%$ level. The correlation between the two time questions in this experiment is equal to .61, also highly significant. 
Imp. Lot. 2 Imp. Lot. 3

\begin{tabular}{ccc}
\hline \hline Imp. Lottery 1 & $.43^{\star \star \star}\left(.37^{\star \star \star}\right)$ & $.36^{\star \star \star}\left(.32^{\star \star \star}\right)$ \\
Imp. Lottery 2 & 1 & $.49^{\star \star \star}\left(.47^{\star \star \star}\right)$ \\
\hline
\end{tabular}

$\star \star \star$ : significant at $1 \%$.

Table 3: Experiment 2 (web) . Correlation among the three imprecision questions. 400 subjects. In parenthesis, correlation without the extreme subjects (365 subjects).

The general lesson here is that the correlation among questions pertaining to a particular domain is in general pretty high. This tends to prove that subjects were consistent in their answers and paid some attention to what they were doing.

\section{Determinants of risk, imprecision and time attitude}

We will not engage in a thorough study of the determinants of the three attitudes studied. We find rather inconclusive results on the general determinants of risk, imprecision and time attitudes. Simple regressions of our measures on age, sex, ${ }^{10}$ income and education level do not yield very significant results (as in most of the literature, where unobserved heterogeneity and noise is known to plague the data), especially when we look at the non extreme population.

We therefore relate two facts that are, to the best of our knowledge, new in the literature. The first concerns gender differences in time attitude. The second concerns the determinant of extremely cautious behavior in the risk and imprecision domain.

\subsection{Impatience by gender}

Table 4 shows that there is very little difference in our measures by gender except for impatience.

We do find that men are more patient than women in the student population but not in general population. However, this needs to be qualified as we did not ask the same time questions in the controlled experiment and in the web experiment. In the latter, there was no question today vs future, but the two questions were future vs more distant future.

Table 5 reports the number of delayed choices by gender for each question in the three experiments. We see that the gap between men and women answers is highest for the first question that involved a choice of today vs. a month.

The previous finding points out a difference in the treatment of the present vs. future by men and women. We report next the significance level of a Mann-Whitney test, where $\mathrm{H}_{0}$ is the equality of the variable NDelayed for men and women. This hypothesis can be rejected at the $10 \%$ level for the overall measure in experiment 1 and 3 . The rejection is "solely" due to the significant differences in their attitude of the present vs future.

Table 6 leads to think that there is a significant difference between men and women in their attitude toward present versus future, but not in their attitude toward future versus more distant

\footnotetext{
${ }^{10}$ See Eckel and Grossman (2008) for an extensive discussion of gender effects on risk aversion.
} 
Experiment $1 \quad$ Experiment $2 \quad$ Experiment 3

(students only)

\begin{tabular}{cccccccc}
\cline { 7 - 8 } Gender & $\mathrm{F}$ & $\mathrm{M}$ & $\mathrm{F}$ & $\mathrm{M}$ & $\mathrm{F}$ & $\mathrm{M}$ \\
\# subjects & $(86)$ & $(78)$ & $(229)$ & $(171)$ & $(46)$ & $(38)$ \\
\hline \hline NCertain & 17.31 & 17.6 & 19.86 & 19.6 & .20 & .17 \\
NPrecise & 18.02 & 18.4 & 14.14 & 14.36 & 31.34 & 31.57 \\
NDelayed & 12.48 & 15.1 & 9.37 & 9.7 & 12.46 & 15 \\
\hline
\end{tabular}

Table 4: Gender effect on risk, imprecision and time attitude. For experiment 3, the measure of risk aversion is the average relative risk premium -see section 6 .

\begin{tabular}{|c|c|c|c|c|c|c|}
\hline & \multicolumn{2}{|c|}{ Now vs 1 month } & \multicolumn{2}{|c|}{1 vs 2 months } & \multicolumn{2}{|c|}{1 vs 7 months } \\
\hline & $\mathrm{F}$ & M & $\mathrm{F}$ & M & $\mathrm{F}$ & M \\
\hline Expe. 1 & 4.16 & 5.35 & 3.95 & 4.7 & 4.36 & 25.04 \\
\hline Expe. 2 & $\mathrm{xx}$ & $\mathrm{xx}$ & 4.47 & 4.42 & 4.91 & 5.3 \\
\hline Expe. 3 (students) & 3.8 & 5.1 & 3.24 & 4.5 & 5.4 & 6.1 \\
\hline
\end{tabular}

Table 5: Average number of delayed choices by gender.

future. Thus, women appear to be more impulsive than men in our experiments. van Praag and Booij (forthcoming) also finds that men are more patient in their sample.

\section{$4.2 \quad$ Extremes}

A striking feature of the graphs of NCertain and NPrecise is the presence of extremely cautious individuals (that are not present in the student population.) It is not clear to us where this extreme behavior comes from. It might be that subjects avoided thinking hard about the choices asked and opted for the "easiest answer"; or it might be that subjects did not pay attention and opted for the fastest way to answer which was (possibly) to always select the right column in those questions; or it might be that subjects were not very numerate and felt uneasy with these unusual questions, or it might simply reflect a truly very large dislike for any uncertainty...

We are not able, given our data, to finely distinguish among these possible explanations. We simply report in table 7 the result of logistic regressions in which the dependent variable was whether the subject is "extreme" or not in each domain and the independent variables are age, sex, level of study (divided into 9 categories) and income (divided into thirteen brackets.) We also report the same logistic regression with an extra dummy, Irr_lottery. This dummy is equal to 1 if subjects gave a higher willingness to pay for a hypothetical lottery ticket that pays 20 euros in case of success compared to a lottery ticket that pays 5000 euros in case of success. These questions were included in the questionnaire subjects had to send back and are essentially the only way we have to "test" subjects' understanding of lottery type of questions. It could also 


\begin{tabular}{ccccc} 
& NDelayed & Q1 & Q2 & Q3 \\
\hline \hline Expe. 1 & $10 \%$ & $3.7 \%$ & $18.7 \%$ & $32 \%$ \\
Expe. 2 & $63 \%$ & $\mathrm{xx}$ & $68 \%$ & $35 \%$ \\
Expe. 3 (students) & $9 \%$ & $10 \%$ & $13 \%$ & $38 \%$ \\
\hline
\end{tabular}

Table 6: Significance level for Mann-Whitney test of equality of answers to time questions by sex.

reflect the fact that some subjects did not pay much attention to their answer. Note however that in their study of truck drivers, Burks, Carpenter, Gotte, and Rustichini (2008) also find that some subjects always chose the safe option and that the "average IQ among those who always prefer the sure payment is one standard deviation below those who behave in a risk neutral way".

\begin{tabular}{ccccc} 
& $\begin{array}{c}\text { Extreme } \\
\text { (risk) }\end{array}$ & $\begin{array}{c}\text { Extreme } \\
\text { (imprecision) }\end{array}$ & $\begin{array}{c}\text { Extreme } \\
\text { (both) }\end{array}$ & $\begin{array}{c}\text { Extreme } \\
\text { (both) }\end{array}$ \\
\hline \hline Age & $.045^{\star \star}$ & $.016^{\star}$ & $.04^{\star \star}$ & $.03^{\star \star}$ \\
& $(.001)$ & $(.1)$ & $(.012)$ & $(.034)$ \\
Sex & .3 & .17 & -.14 & -.009 \\
& $(.4)$ & $(.54)$ & $(.71)$ & $(.98)$ \\
Income & -.08 & -.07 & -.11 & -.089 \\
& $(.18)$ & $(.22)$ & $(.14)$ & $(.28)$ \\
Education & $-.18^{\star}$ & $-.16^{\star \star}$ & $-.24^{\star \star}$ & $-.32^{\star \star \star}$ \\
& $(.08)$ & $(.05)$ & $(.04)$ & $(.009)$ \\
Irr_lottery & & & & $.75^{\star}$ \\
& & & & $(.06)$ \\
\hline Obs. & 386 & 386 & 386 & 364 \\
Wald chi2 & 25.55 & 11.1 & 18.26 & 24.3 \\
Prob>Chi2 & 0 & .026 & .001 & .0002 \\
Pseudo $\mathrm{R}^{2}$ & .093 & .032 & .087 & .11 \\
\hline
\end{tabular}

In parenthesis, significance level (p-values).

$\star$ significant at $10 \%, \star \star$ : significant at $5 \%, \star \star \star$ : significant at $1 \%$.

Table 7: Web experiment. Logistic regressions.

A conclusion that can be drawn from this table is that elder people tend to be extremely cautious, while more educated people will have lesser chance to behave in an extreme manner vis-à-vis risk and imprecision. Our proxy for "cognitive ability" (in a very partial sense, since it could be also a measure of their attention while filling in the questionnaire and doing the experiment subsequently) is also significant. ${ }^{11}$ Income is not significant.

We did the same regressions including wealth in the regressors. The decay in sample size

\footnotetext{
${ }^{11}$ This would be in line with findings in Burks, Carpenter, Gotte, and Rustichini (2008) who have a much more precise and well defined notion of cognitive ability and show it is strongly relevant to explain subjects risk and time preferences. This is also compatible with findings reported in Dohmen, Falk, Huffman, and Sunde (2008).
} 
is quite large as we have only 354 subjects left (only 360 out of our 400 subjects answered the question related to their wealth). Wealth is significant in the risk part (with a negative sign as could be expected). Surprisingly, income then becomes significant with a positive sign. Wealth is not significant to explain extreme behavior along the imprecision dimension.

\section{Correlations}

In this section, we look at correlations of answers across domains. In the web experiment, we find a significant positive correlation between risk and imprecision attitudes (table 8) while the other correlations are equal to 0 . However, the correlation between NCertain and NPrecise is entirely driven by the presence of extreme subjects, as can be seen in Figure 4.

\begin{tabular}{ccc} 
& NPrecise & NDelayed \\
\hline \hline NCertain & $.22^{\star \star \star}$ & .002 \\
& $(.00)$ & $(.97)$ \\
NPrecise & 1 & -.05 \\
& & $(.28)$ \\
\hline
\end{tabular}

In parenthesis, significance level.

$\star \star \star$ : significant at $1 \%$.

Table 8: Web experiment. Correlation between the three measures. 400 subjects.

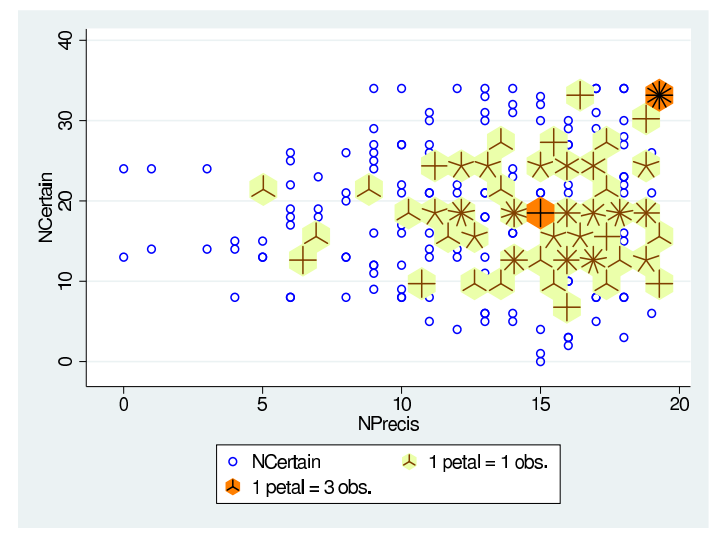

Figure 4: Web experiment. NCertain vs. NPrecise

Indeed, if we drop these subjects from our sample, the correlation between NCertain and NPrecise disappears, as reported in Table 9.

Finally, we checked whether the results reported here are due to the presence of non monotonic agents in the sample. This is not the case since when we use the sub sample of monotonic agents we find similar results. The significant correlation between NPrecise and NCertain is again exclusively due to the presence of extreme subjects.

As could be expected then, in experiment 1, run mostly on students and in which we did not 


\begin{tabular}{lcc} 
& NPrecise & NDelayed \\
\hline \hline NCertain & $\mathbf{. 0 4}$ & .00 \\
& $(.42)$ & $(.99)$ \\
NPrecise & 1 & -.06 \\
& & $(.22)$ \\
\hline In parenthesis, significance level.
\end{tabular}

Table 9: Web experiment. Correlation between the three measures. 365 (not extreme) subjects.

observe the presence of extreme subjects, the correlation between risk and imprecision is also not significantly different from zero.

\section{A more focussed protocol and the contraction model}

\subsection{Description}

The third experiment was run at the experimental laboratory of University Paris I on 88 "usual" subjects (mostly students) and 16 portfolio managers. The experimental protocol was modified compared to the one reported above and bore only on risk, imprecision and time attitudes (we will not comment on the latter since this was already done in section 3.3.) The risk design was made so as to minimize the number of questions asked to elicit a certainty equivalent of a given lottery in order to collect more information. For instance, a decision maker who has shown strong risk aversion on previous questions was not subsequently asked to choose between a lottery and its expected value. In that way, we elicited the certainty equivalent for 15 different lotteries with roughly 50 choices only. These lotteries were of the form win $x(x=20,50,80)$ with probability $p(p=.1, .3, .5, .7, .9)$ and win nothing otherwise.

A drawback of this approach is that it assumes a lot of internal consistency on the subjects. If we determined for instance that a subject had a high degree of risk aversion in the first questions, then we would ask this subject to choose only between the lottery $(20, .5 ; 0, .5)$ and $4,5,6$, or 7 euros for sure for instance. If he switched between choosing the lottery and a certain amount within this interval, then we have a good estimate of his certainty equivalent. On the other hand, if he always chose the lottery, the only information we have is that his certainty equivalent is higher than 7. Conversely if he always chose the certain amount, we know only that his certainty equivalent is smaller than 4 . Thus, we do not have estimate of certainty equivalents for these lotteries but only an upper or lower bound. Most subjects hit these bounds at least for one or two lotteries out of the fifteen. ${ }^{12}$

\subsection{Measure of risk aversion}

To get an aggregate measure of risk aversion we computed the relative risk premium $\frac{1}{n} \sum_{i}\left(E_{i}-\right.$ $\left.E C_{i}\right) / E_{i}$ where $E C_{i}$ is the certainty equivalent for lottery $i$ and $E_{i}$ its expected value and

\footnotetext{
${ }^{12} 4$ subjects hit the bounds on all 15 questions and were consequently dropped.
} 
$n$ is the number of lotteries for which bounds were not hit. The mean average relative risk premium is equal to $.18(\mathrm{std}=.23-$ median $=-.21)$ thus showing risk aversion on the aggregate. Not surprisingly, the average relative risk premium is significantly higher for students (.186) than for portfolio managers (.146).

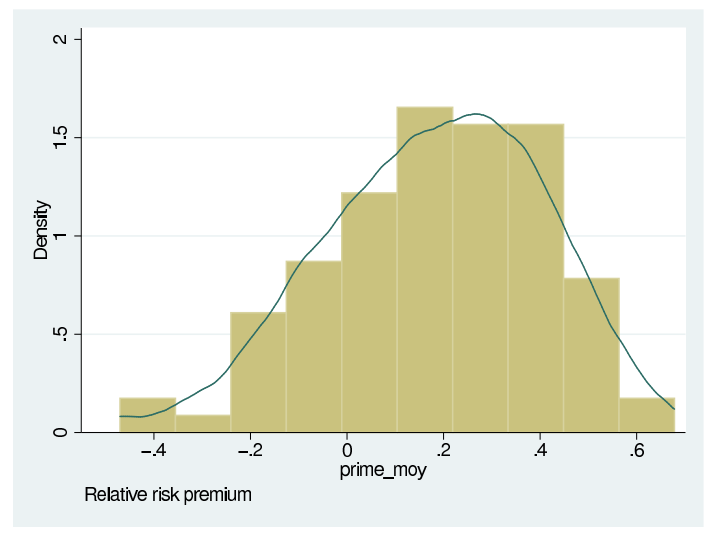

Figure 5: Relative risk premium, 100 subjects (students and portfolio managers. The 4 subjects that hit the bounds were dropped.)

\subsection{Measure of imprecision aversion}

In the imprecision questions, the subjects were again asked to choose between precise and imprecise lotteries. The only change with respect to the previous protocol was that for each series the imprecise lottery was kept fixed while the odds for the precise lottery were changing. Table 13 in the Appendix shows an instance of a series of questions. Contrary to the previous two experiments, the protocol here did not force subjects to behave in an imprecision averse manner and allowed a more precise estimation of their attitude toward imprecision. In particular, the computation of the degree of imprecision aversion as developed in Gajdos, Hayashi, Tallon, and Vergnaud (2008) is almost immediate for this protocol.

In this model, the utility of an imprecise lottery yielding a gain $x$ with unknown probability $p \in[a, b]$ is equal to (normalizing $u(0)=0$ )

$$
\alpha \min _{p \in[a, b]} p u(x)+(1-\alpha)\left(\frac{a+b}{2}\right) u(x)
$$

that is, $\alpha a u(x)+(1-\alpha)\left(\frac{a+b}{2}\right) u(x)$. In this formula, $\alpha$ is an index of imprecision aversion. It is equal to 0 when the subject behaves as an expected utility maximizer (with beliefs equal to the median probability of success) and equal to 1 if the subject only considers the lowest probability of success.

Our experimental data can be used to compute $\alpha$ in a straightforward manner. For each series of question, we assess the probability at which the subject switched from the precise lottery to the imprecise lottery. At this probability (call it pswitch), the subject is indifferent among the 
two options and hence

$$
\alpha . a . u(x)+(1-\alpha)\left(\frac{a+b}{2}\right) u(x)=\operatorname{pswitch} . u(x)
$$

The utility of the gain $u(x)$ simplifies out and we can thus compute

$$
\alpha=\frac{\frac{a+b}{2}-p s w i t c h}{\frac{b-a}{2}}
$$

Note this formula applies only for imprecision averse subjects, whose switching probability is less than the median unknown probability. Higher $\alpha$ reflects more imprecision averse choices. ${ }^{13}$ This is independent of the utility function and two decision makers can be ranked in terms of imprecision aversion regardless of their utility index $u$. This separation of imprecision attitude from risk attitude is a general feature of the contraction model of Gajdos, Hayashi, Tallon, and Vergnaud (2008). A decision maker with a higher $\alpha$ can unambiguously be said to be more imprecision averse than a decision maker with a lower $\alpha$, even if they have different utility functions. In our experiment, because there are only two possible outcomes, this is also true of the $\alpha$-MMEU model.

In our data, the switching point is not estimated very precisely. For instance, if we consider a subject facing the series of choices as described in Table 13 in the Appendix. Imagine that this subject chose option $\mathrm{A}$ in his first three choices and then chose option $\mathrm{B}$ from choice number 4 onward. He switched from the imprecise to the precise option between probability .3 and probability .4 of winning in the precise lottery. In the statistical analysis, we chose the midpoint .35 as the switching probability. A consequence of this choice is that, in this example, a subject that starts to choose the precise lottery when the chances of winning are .6 or above will be said to be imprecision seeking (his switching probability is equal to .65), while he could well be neutral (his "true" switching probability is equal to .6, the middle of the interval $[.2,1]$.) In the data, we find quite a significant proportion (up to 15\%) of subjects who switched at the probability equal to the middle of the interval.

We computed the coefficient $\alpha$ for each subject and each series of question and then averaged these coefficients across questions to obtain an estimation of the imprecision aversion per subject. ${ }^{14}$ Out of our pool of 104 subjects, 37 did switch for at least one series of question at or above the mean of the probability interval, thus revealing neutrality or optimism in at least one series (the contraction coefficient $\alpha$ is then negative). Only four subjects had a negative average contraction coefficient.

On the whole sample, the mean value for $\alpha$ is .28 (approximately equal to the median) and standard deviation is .18. When we restrict attention to the 67 subjects whose coefficient is positive on all 8 questions, the mean value raises to .36 (also equal to the median) while standard deviation is lower, equal to .15. Imprecision aversion is higher in the student population (mean

\footnotetext{
${ }^{13}$ When there are just two states, as is the case in this study, $\alpha<0$ captures ambiguity seeking behavior.

${ }^{14}$ Given the very simple linear structure of the problem, this amounts to do a least square estimation of $\alpha$ on all 8 questions.
} 


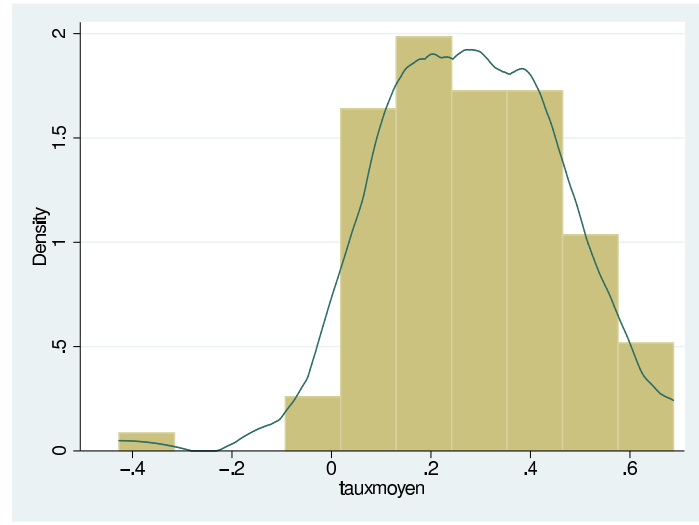

Figure 6: Contraction rate (imprecision aversion), 104 subjects (students and portfolio managers.)

equal to .30) than in the portfolio manager population (mean equal to .18) Overall, we find quite a lot of individual heterogeneity. Contrary to the web experiment however, there are no extreme subjects.

As explained in the Appendix, we asked the same questions to the subjects, for two different levels of payoffs (20 and 50 euros). If we average the coefficient $\alpha$ on the four series of questions with payoff $20 €$ and compare it to the coefficient obtained by averaging over the four series of questions with payoff $50 €$, we find no difference at all. When we test directly, for each information, the equality of the coefficient for the 20 euros question with the one for the 50 euros question, we find equality of the coefficients for three out of four questions. Thus, dependence of the contraction rate on the information, which is allowed in the general formulation of the contraction model of Gajdos, Hayashi, Tallon, and Vergnaud (2008), is not found in the data which tend to confirm the more restrictive case of a constant $\alpha$.

Ahn, Choi, Gale, and Kariv (2009) report estimation of a parameter of "ambiguity aversion" for the $\alpha-M M E U$ case where a decision is evaluated by the weighted average between the minimal and the maximal expected value. In the present setting, this yields a criterion that can be written:

$$
\alpha \cdot a \cdot u(x)+(1-\alpha) \cdot b \cdot u(x)
$$

The $\alpha$-MMEU model in the present setting is thus equivalent to the contraction model. There is a direct relationship between the rate of imprecision aversion we computed and the $\alpha$ of the $\alpha$-MMEU model: $\alpha_{G H T V}=2 \alpha_{M M E U}-1$. Our value of .28 thus translates into $\alpha_{M M E U}=.64$ while Ahn et al. find an average equal to .565. Our estimates are thus comparable to what they found, although the experimental setting is rather different. Potamites and Zhang (2007) report a mean of $\alpha_{M M E U}$ conditional on being ambiguity averse (i.e., conditional on $\alpha_{M M E U}>.5$ ) equal to .68 which is also of comparable magnitude to our measure: when we restrict attention to subjects that are imprecision averse in all series, the corresponding coefficient is .69. 


\subsection{The absence of correlation between risk and imprecision aversion}

We finally report the correlation between the relative risk premium and the coefficient of imprecision aversion. On those subjects with positive average contraction coefficient, the correlation between this coefficient and the relative risk premium is equal to .06, not significantly different from 0 . Hence, here again, we find no correlation between risk and imprecision attitudes.

Remark 1 On the same data set, Roux (2008) estimated -by maximum likelihood- rank dependent models for the risk part. He for instance considered a specification with a power utility function and a two-parameter probability weighting function (one measuring elevation, the other the distortion) due to Prelec. We find a negative significant correlation of the coefficient of the utility function with the contraction coefficient and no correlation of the latter with the two parameters of the weighting function.

\section{Three intriguing facts}

We end this paper by mentioning three "puzzles" detected in our data set, which show the need for further research in the way risk and imprecision attitudes should be elicited.

We used in this paper measures based on controlled experiments that were of a similar format across the three experiments. In the first two experiments, we also have measures based on self reported scales, widely used in psychology. We also asked hypothetical questions such as the by now well known Barsky, Juster, Kimball, and Shapiro (1997) lottery. Finally, in the third experiment we also asked the subjects to make choices in the context of the three color Ellsberg experiment.

In these three cases, we thus had another source of information on subjects' attitude toward risk and uncertainty. ${ }^{15}$ The (maybe not so) surprising result is that these various sources are at best weakly correlated with the measures we used so far in the paper.

\subsection{Scales versus experiments}

In experiment 1 and 2 , subjects also reported on a scale from 0 to 10 their own assessment of how risk averse they are, how patient they are, how impulsive and how far-sighted. ${ }^{16}$

We concentrate on the general population sample (experiment 2) but the pattern is similar in the first experiment. Again somewhat surprisingly, the correlations among these four scales and our experimental measures for the general population are not significant. Spearman's coefficients are also close to 0 . This is true for both the whole sample (400 subjects) and for the sub sample

\footnotetext{
${ }^{15}$ Through the questionnaire subjects filled out we also have a lot of indirect information on their attitudes. Based on the same survey, Arrondel \& Masson used questions that were a priori linked to risk, ambiguity or impatience to build individual scores on each of these dimensions.

${ }^{16} \mathrm{On}$ a scale from 0 to 10 , do you consider yourself as someone who is prudent or conversely as someone who likes to take risk, likes adventure? (...) as someone who is patient or conversely someone who is impatient, in a hurry? (...) as someone thoughtful or conversely as impulsive? (...) as someone who takes every day as it comes or conversely, someone who plans things and is far-sighted?
} 
constituted of only non extreme subjects. When we restrict the sample to monotonic and non extreme subjects there is a significant correlation between NCertain and the risk scale as well as between NDelayed and the scale thoughtful/impulsive. But both these correlations are fairly small (-.18 and -.15 respectively.) Dohmen et al. (2005) do find a significant link between a binary categorization in terms of risk aversion based on a scale and subjects' actual behavior in a lottery. The variance explained by the measure is however fairly small.

We view this finding as calling for more research on the link between self reported scales, widely used in psychology and experimental measures as we use them in economics.

\subsection{Hypothetical lotteries}

In the survey, we asked the Barsky, Juster, Kimball, and Shapiro (1997) hypothetical lottery that allows one to classify decision makers in four categories. We can thus construct a variable that takes value 1 for the most "risk averse" agents and 4 for the less "risk averse". The Spearman coefficient of correlation between NCertain and this variable is equal to -.12 (significant at $5 \%$ ) on the full sample and to -.13 (also significant) on the non extreme subjects. While the correlation is significant and of the good sign, it is fairly small and again points to the "multi-dimensionality" of the attitude we want to measure. The correlation with NPrecise is equal to zero in both cases.

We also asked hypothetical questions (willingness to pay for a lottery ticket that pays 20 (resp. 5000) euros or zero with probability .5). Here also, the answers have essentially zero correlation with our measures of risk and imprecision aversion (both on the entire sample and the non extreme sample.)

\subsection{Two urns versus three colors}

In experiment 3, we asked the subjects to answer a "three color Ellsberg" type of question. Subjects could choose among decisions involving bets on the color of a marble drawn from an urn in which there are 30 red marbles and 60 black or white marbles in unknown proportion. Specifically, they were asked to choose an option among A, B, C in Table 10, and then among D, E, F.

\begin{tabular}{cccc} 
& Red & Black & White \\
\hline \hline A & 20 & 0 & 0 \\
B & 0 & 20 & 0 \\
C & 0 & 0 & 20 \\
\hline \hline D & 20 & 20 & 0 \\
E & 20 & 0 & 20 \\
F & 0 & 20 & 20
\end{tabular}

Table 10: Decision matrix. Three color Ellsberg urn.

The subjects were presented with these symmetric choices to avoid fear of manipulation concerning the composition of the urn from which they drew (if the question was selected among 
all the experimental questions.) The typical choice predicted by Ellsberg is to choose A in the first situation and F in the second, thus revealing "ambiguity aversion" of a type that is incompatible with expected utility (incompatible with the sure thing principle for that matter.) ${ }^{17}$

Out of the 104 subjects, 39 subjects only chose (A,F), which still constitutes the modal choice. (A,D) was chosen by 22 subjects and $(\mathrm{B}, \mathrm{F})$ by 17 subjects. Given the evidence in favor of imprecision aversion found in the imprecise lottery versus precise lottery questions, it is surprising that only 39 subjects chose according to what imprecision aversion would dictate.

When we want to explain the Ellsbergian choice $(\mathrm{A}, \mathrm{F})$ by running a logit regression on the imprecision aversion coefficient, we obtain contrasted results, reported in Table 11. The coefficient of imprecision aversion is not significant while the dummy "optimist", that is equal to 1 if the subject has, in at least one series of question, chosen in an imprecision neutral or seeking way, is highly significant. Risk aversion however does not play any role, which is reassuring.

\begin{tabular}{c|cccc} 
& \multicolumn{4}{|c}{ Ellsberg } \\
\hline \hline Imp. aversion & 2.02 & & -.01 & .40 \\
& $(.113)$ & & $(.99)$ & $(.80)$ \\
Optimist & & $-1.41^{\star \star \star}$ & $-1.41^{\star \star}$ & $-1.29^{\star \star}$ \\
& & $(.006)$ & $(.02)$ & $(.034)$ \\
Av. premium & & & & -.061 \\
& & & & $(.95)$ \\
\hline \hline obs. & 100 & 100 & 100 & 97 \\
Wald chi & \\
& 2.50 & 7.52 & 7.56 & 7.33 \\
Proba $>\mathrm{chi}^{2}$ & .11 & .006 & .023 & .0621 \\
Pseudo $\mathrm{R}^{2}$ & .019 & .066 & .066 & .062 \\
\hline \hline
\end{tabular}

In parenthesis, significance level.

$\star \star$ : significant at $5 \%, \star \star \star$ : significant at $1 \%$.

Table 11: Logit regression of the variable Ellbserg ( $=1$ if the subject chose (A,F), 0 otherwise.) Constant omitted. Imprecision aversion is the mean contraction coefficient over the 8 series of questions.

The variable "optimist" is based on a categorization that is pretty qualitative, contrary to the imprecision aversion coefficient which is by nature quantitative. Yet, the average imprecision coefficient among the "optimistic subjects" (mean=.17, std=.097) is much lower than for non optimistic subjects $($ mean $=.36, \mathrm{std}=.15)$. However, it is not this quantitative aspect that seems to be instrumental to explain behavior in the three color Ellsbergian urn.

\section{Conclusion}

The experimental results reported in the present paper are rather stark. Subjects' behavior is virtually independent across the three domains studied, when we account for the existence

\footnotetext{
${ }^{17}$ Notice that in this "symmetrized" version of the three color urn, answers (B,E) and (C,D) are also incompatible with the sure thing principle (but could arguably be said to be irrational given the information available.)
} 
of extreme subjects. This gives argument, we feel, in favor of developing models in which 1) imprecision attitudes is not reduced to risk attitudes and 2) there is a complete separation between these two concepts. Hence, this study tends to reinforce the idea that we need at least three parameters (risk aversion, imprecision aversion and impatience) to attempt to describe and predict any decision maker's choice. In a companion paper we aim to exploit the distinction between the three attitudes and show how each dimension can be related to a particular feature of subject's financial behavior. This study also showed an important different feature between the student population and the general population, namely the presence of extreme subjects in the latter, that tend to avoid any form of risk. Exploring the robustness of this finding (was it only due to the fact that the experiment was on line?) and relating it to measure of cognitive abilities seems like an interesting avenue for further research. 


\section{APPENDIX}

\section{Experimental protocols}

\subsection{Experiment 1}

This experiment was run at University Paris I, on 164 subjects (students for the most part) and was part of a larger project. It was run during April 2007. Subjects came twice. On their first visit, they filled out a long questionnaire (it took between an hour and a half and two hours to complete). This questionnaire asked information about their behavior in various domains (health, consumption, leisure, finance...) as well as background information. Subjects subsequently came back to the experimental lab for a session that lasted about an hour. In this session, they played a usual dictator game, a trust game, a provision of public good game, a modified dictator game. They also answered three (series of) "risk questions", five (series of) "imprecision questions" and three (series of) "time questions". All the experiment was computerized but for the time questions which were answered on a separate sheet of paper. One question (outside of the time questions, see below) was drawn at random for each participant. Subjects were paid according to the answer they gave to that question.

\subsubsection{Risk, imprecision and impatience}

The risk questions were framed as follows: do you prefer a gain of $z$ euros contingent on drawing a winning ball from a 10-ball urn that contains exactly $x$ winning balls or a sure gain of $w$ euros? $z$ was equal to 20 when $x=5,30$ when $x=3$ and 15 when $x=7$. The sure gain of $w$ euros was set alternatively at a high and a low value (according to the so-called bracketing technique). Payment for this question, if it involved playing a lottery, was done as follows: the subject would choose a winning color (black or white) and the experimenter would then fill an urn with the appropriate number of winning and losing marbles. The subject then drew a marble, which determined its payment.

The imprecision questions were framed as follows: do you prefer a gain of 20 euros contingent on drawing a winning ball from a 10 ball urn that contains between $a$ and $b$ winning balls or a gain of 20 euros contingent on drawing a winning ball from a 10 ball urn that contains exactly $x$ winning balls? There were fives series of such questions. The first three had $x=5$ and $a$ or $b$ changing. In the first series, $b$ is fixed at 10 and $a$ goes from 0 to 6 ; in the second, $b$ is fixed at 8 and $a$ goes from 0 to 5; in the third, $a$ is fixed at 2 and $b$ goes from 10 to 5 . The fourth series had $x=3, a=2$ and $b$ going from 10 to 4 . The fifth series had $x=7, a$ going from 2 to 7 and $b=10$.

Thus, for a given series (say the second series) a subject had to make choices that can be represented as in table 12 . Note that this was not the table subjects were filling, as each question was asked one after the other. Subjects in the lab had the history of their answers for each series of question on the screen. Subjects in the web experiment did not have this information.

\subsubsection{Payment scheme: how to implement imprecise lotteries and delayed rewards}

Payment for these questions was done as follows. To avoid fear of manipulation, subjects decided themselves which color was the winning color and subsequently drew from the chosen urn. More precisely, the experimenter showed an urn with 10 marbles already in it (that the subject could not see). If the composition of the urn was said to be "between $a$ and $b$ winning balls", the experimenter would take out of the urn $a+(10-b)$ marbles. He would then ask the subject 


\begin{tabular}{|c|cc|cc|}
\hline & \multicolumn{2}{|c|}{ Option A } & \multicolumn{2}{c|}{ Option B } \\
\hline \hline Question & $\begin{array}{c}\text { Information } \\
\text { on the urn }\end{array}$ & $\begin{array}{c}\text { Gain in case } \\
\text { of success }\end{array}$ & $\begin{array}{c}\text { Information } \\
\text { on the urn }\end{array}$ & $\begin{array}{c}\text { Gain in case } \\
\text { of success }\end{array}$ \\
\hline 1 & $\begin{array}{c}\text { Between } 0 \text { and } 8 \\
\text { winning balls }\end{array}$ & $20 €$ & $\begin{array}{c}\text { Exactly } 5 \\
\text { winning balls }\end{array}$ & $20 €$ \\
\hline 2 & $\begin{array}{l}\text { Between } 1 \text { and } 8 \\
\text { winning balls }\end{array}$ & $20 €$ & $\begin{array}{c}\text { Exactly } 5 \\
\text { winning balls }\end{array}$ & $20 €$ \\
\hline 3 & $\begin{array}{l}\text { Between } 2 \text { and } 8 \\
\text { winning balls }\end{array}$ & $20 €$ & $\begin{array}{l}\text { Exactly } 5 \\
\text { winning balls }\end{array}$ & $20 €$ \\
\hline 4 & $\begin{array}{l}\text { Between } 3 \text { and } 8 \\
\text { winning balls }\end{array}$ & $20 €$ & $\begin{array}{c}\text { Exactly } 5 \\
\text { winning balls }\end{array}$ & $20 €$ \\
\hline 5 & $\begin{array}{l}\text { Between } 4 \text { and } 8 \\
\text { winning balls }\end{array}$ & $20 €$ & $\begin{array}{l}\text { Exactly } 5 \\
\text { winning balls }\end{array}$ & $20 €$ \\
\hline 6 & $\begin{array}{c}\text { Between } 5 \text { and } 8 \\
\text { winning balls }\end{array}$ & $20 €$ & $\begin{array}{c}\text { Exactly } 5 \\
\text { winning balls }\end{array}$ & $20 €$ \\
\hline
\end{tabular}

Table 12: Binary choices in the second series of imprecision questions. Experiments 1 (students) and 2 (general population).

which winning color he chose (black or white) and then put back in the urn $a$ marbles of the winning color and $10-b$ marbles of the losing color. The subject thus knew that the information given to them was reliable and that the experimenter could not manipulate the composition of the urn in advance since he did not know the choice of the winning color by the subject. The subject then drew a marble from the urn and payment was made accordingly.

The three time questions were on the format "do you prefer 100 euros at a given date or $100+x$ at a later date time?". In the first series, the two dates were now and in one month and $x$ went from 0 to 20 (by increment of 2). In the second series, the dates were one month and two months from now and $x$ went from 0 to 20 (by increment of 2). In the third series, the dates were one month and seven months from now and $x$ went from 0 to 50 (by increment of 5). Payment was done as follows. In each experimental session (that is, for every eighteen subjects), one subject was also paid on the basis of his choices in this part. For this subject, one question was picked at random and the subject paid accordingly. If it involved payment at a later date, the experimenter asked the subject what was the most convenient time (within a few days around the exact term) and place (within Paris) to meet to actually deliver the payment. It involved, for a few subjects, delivering cash at their place. The experimenter signed a receipt. This procedure was aimed at minimizing (for the subject) as much as possible transaction costs associated to delayed payments.

\subsection{Experiment 2: web experiment}

\subsubsection{A "representative" population}

The second experiment is almost identical to the first one except that it was run on the web on a sample of 400 people from the general French population. In this study a survey questionnaire, asking information about behavior in various domains (health, consumption, leisure, finance...) as well as background information, was sent, through the poll institute TNS-Sofres to a sample of the French population, consisting of 4000 persons. We got back 3826 questionnaires. 
Participants were asked at the end of the questionnaire if they were willing to participate to a follow-up that would be implemented via the web, in which case they had to give an e-mail address. A couple months after we got back all the questionnaires, TNS-Sofres sent an e-mail to all the respondents who had given an address, giving the participants the URL address of a web site. This web site was taking them through an on line experiment. The experiment was about 20 minutes long. The first 400 connected people did the experiment. The entire process (sending messages and collecting the 400 experimental data) took less than 2 days. The experiment was run during the spring 2007.

\subsubsection{Design and payment scheme}

The experiment was roughly the same as the one described in the previous section, only it was made shorter. The questions of interest for us here are the same three risk questions, the first three imprecision questions and the last two time questions.

Subjects could click on a button that was explaining how payments would be made. For the risk questions, payment was implemented by a computerized random draw that was made in front of a representant of TNS-Sofres ${ }^{18}$ The payment scheme implemented for imprecision questions in experiment 1 was not implementable on the web. We replaced it with the following scheme: if the question involved drawing from an urn in which there are between $a$ and $b$ winning balls, a number $s$ was drawn at random (according to a uniform law on [0,1]). The first digit of $s^{2}$ that fell in the interval $[a, b]$ was then picked to determine the composition of the urn. For instance, if $s^{2}=.1739585, a=$ and $b=6$, the urn from which the draw was made had 3 winning balls since 3 is the first digit of $s^{2}$ that is consistent with the information given.

Finally, we asked only two time questions, in which the subjects had the choice between a 100 euros payment in a month and $100+x$ euros in two months (resp. seven months), as immediate payment was not feasible. The probability for the time questions to be picked was set so that it mimicked the scheme implemented for the students.

Payments were made by TNS-Sofres (with the corresponding delay for the time questions) which sent gift certificates to the participants (those had values in well above 100 big French stores). Respondents earned on average 17.5 euros for the experimental phase.

\subsection{Experiment 3: students and portfolio managers}

The third experiment was run at the University Paris I on 88 "usual" subjects (mostly students) and 16 portfolio managers and support staff. Sessions took place in the spring 2008.

\subsubsection{A more focussed protocol}

The experimental protocol was modified compared to the first two experiments reported here. ${ }^{19}$ The experiment bore only on risk, imprecision and time attitudes (and not on behavior in games as it was the case in the two previous experiments.) The risk design was made so as to minimize the number of questions asked to elicit a certainty equivalent of a given lottery (in order to be able to increase the collected information.)

\footnotetext{
${ }^{18}$ This institute was at first reluctant to implement random payments, as it might have been associated with gambling behavior which was, until recently, very heavily regulated in France, the state having a monopoly in that domain.

${ }^{19}$ See Roux (2008).
} 


\begin{tabular}{|c|c|c|c|c|}
\hline & \multicolumn{2}{|c|}{ Option A } & \multicolumn{2}{|c|}{ Option B } \\
\hline Question & $\begin{array}{l}\text { Information } \\
\text { on the urn }\end{array}$ & $\begin{array}{c}\text { Gain in case } \\
\text { of success }\end{array}$ & $\begin{array}{l}\text { Information } \\
\text { on the urn }\end{array}$ & $\begin{array}{l}\text { Gain in case } \\
\text { of success }\end{array}$ \\
\hline 1 & $\begin{array}{c}\text { Between } 2 \text { and } 10 \\
\text { winning balls }\end{array}$ & $20 €$ & $\begin{array}{c}\text { Exactly } 2 \\
\text { winning balls }\end{array}$ & $20 €$ \\
\hline 2 & $\begin{array}{c}\text { Between } 2 \text { and } 10 \\
\text { winning balls }\end{array}$ & $20 €$ & $\begin{array}{c}\text { Exactly } 3 \\
\text { winning balls }\end{array}$ & $20 €$ \\
\hline 3 & $\begin{array}{c}\text { Between } 2 \text { and } 10 \\
\text { winning balls }\end{array}$ & $20 €$ & $\begin{array}{c}\text { Exactly } 4 \\
\text { winning balls }\end{array}$ & $20 €$ \\
\hline 4 & $\begin{array}{c}\text { Between } 2 \text { and } 10 \\
\text { winning balls }\end{array}$ & $20 €$ & $\begin{array}{c}\text { Exactly } 5 \\
\text { winning balls }\end{array}$ & $20 €$ \\
\hline 5 & $\begin{array}{c}\text { Between } 2 \text { and } 10 \\
\text { winning balls }\end{array}$ & $20 €$ & $\begin{array}{c}\text { Exactly } 6 \\
\text { winning balls }\end{array}$ & $20 €$ \\
\hline 6 & $\begin{array}{c}\text { Between } 2 \text { and } 10 \\
\text { winning balls }\end{array}$ & $20 €$ & $\begin{array}{c}\text { Exactly } 7 \\
\text { winning balls }\end{array}$ & $20 €$ \\
\hline 7 & $\begin{array}{c}\text { Between } 2 \text { and } 10 \\
\text { winning balls }\end{array}$ & $20 €$ & $\begin{array}{c}\text { Exactly } 8 \\
\text { winning balls }\end{array}$ & $20 €$ \\
\hline
\end{tabular}

Table 13: Binary choices in the second series of imprecision questions. Experiment 3.

\subsubsection{Optimized questions}

The protocol started by eliciting the vNM utility function using the trade off method of Wakker and Deneffe (1996). The next questions which elicited certainty equivalent through bisection, were used to get an idea of the probability transformation of the subject. These two pieces of information (an estimate of the vNM utility function and an estimate of the shape of the probability transformation function) were then used to tailor the remaining questions so as to ask the subject to make choices between a lottery and only of few certain amounts close to the estimated (on the basis of the previous information) certainty equivalent. In that way, the certainty equivalent to 15 different lotteries could be elicited with roughly 50 choices only. These lotteries were of the form win $x(x=20,50,80)$ with probability $p(p=.1, .3, .5, .7, .9)$ and win nothing otherwise.

\subsubsection{Imprecision and Ellsberg}

In the imprecision questions, the subjects were again asked to choose between precise and imprecise lotteries. The only change w.r.t. the previous protocol was that for each series the imprecise lottery was kept fixed while the odds for the precise lottery were changing. For instance, the subject was asked which alternative he preferred between a bet with between .2 and .8 chances of winning and a lottery with $p$ chances of winning and $p$ was varied from .1 to .9 say. We had 8 series of questions: the information for the probability of winning for the imprecise lottery was $[0,10],[2,8],[2,10]$, and $[5,10]$ and we asked questions for two levels of gain, 20 and 50 euros.

A typical series of question was thus of the kind represented in table 13

The three time questions were the same as in the first protocol, except that due to time and budget constraint, they were not included in the questions susceptible to give rise to payment.

Finally, we asked the subjects to answer a "three color Ellsberg" type of question. Subjects were told that they have to make choices among two decisions involving bets on the color of a marble drawn from an urn in which there are 30 red marbles and 60 black or white marbles in 
unknown proportion.

They were then asked to chose an option among A,B, C in table 10 and then among D, E, F. The subjects were presented with these symmetric choices to avoid fear of manipulation concerning the composition of the urn from which they drew (if the question was selected among all the experimental questions.)

The typical choice predicted by Ellsberg is to choose A in the first situation and $\mathrm{F}$ in the second, thus revealing "ambiguity aversion" of a type that is incompatible with expected utility (incompatible with the sure thing principle for that matter.) Notice that in this "symmetrized" version of the three color urn, answers (B,E) and (C,D) are also incompatible with the sure thing principle. Subjects also had to answer to a short questionnaire. 


\section{References}

Ahn, D., S. Choi, D. Gale, and S. Kariv (2009): "Estimating Ambiguity Aversion in a Portfolio Choice Experiment," Working paper.

Andersen, S., J. Fountain, G. Harrison, and E. Rutström (2009): "Estimating Aversion to Uncertainty," mimeo.

Andersen, S., G. Harrison, M. Lau, and E. Rutström (2008): "Eliciting risk and time prefrences," Econometrica, 76, 583-618.

Barsky, R., T. Juster, M. Kimball, and M. Shapiro (1997): "Preference parameters and individual heterogeneity: an experimental approach in the health and retirement study," Quarterly Journal of Economics, 112, 537-579.

Burks, S., J. Carpenter, L. Gotte, and A. Rustichini (2008): "Cognitive skills explain economic prefrences, strategic behavior, and job attachment," Discussion paper 3609, IZA.

Cabantous, L. (2007): "Ambiguity aversion in the field of insurance: insurer's attitude to imprecise and conflicting probability estimates," Theory and Decision, 62, 219-240.

Chakravarty, S., and J. Roy (2009): "Recursive expected utility and the separation of attitudes towards risk and ambiguity: an experimental study," Theory and Decision, 66(3), $199-228$.

Chow, C., and R. SARIn (2002): "Known, unknown and unknowable uncertainties," Theory and Decision, 52, 127-138.

Cohen, M., J.-Y. JAFFray, And T. SAID (1987): "Experimental comparison of individual behavior under risk and under uncertainty for gains and for losses," Organizational Behavior and Human Decision Processes, 39, 1-22.

Collard, F., S. MukerJi, K. Sheppard, and J.-M. Tallon (2009): "Ambiguity and the historical equity premium," mimeo.

Curley, S., and F. Yates (1985): "The center and range of the probability interval as factors affecting ambiguity preferences," Organizational Behavior and Human Decision Processes, 36, $272-287$.

Di Mauro, C., And A. Maffioletti (2004): "Attitudes to risk and attitudes to uncertainty: experimental evidence," Applied Economics, 36, 357-372.

Dohmen, T., A. Falk, D. Huffman, and U. Sunde (2008): "Are risk aversion and impatience related to cognitive ability?," Discussion paper 6852, CEPR.

Dohmen, T., A. Falk, D. Huffman, U. Sunde, J. Schupp, and G. Wagner (2005): "Individual risk attitudes: new evidence from a large, representative, experimentally validated survey," Discussion paper 1730, IZA.

Eckel, C., and P. Grossman (2008): "Men, women and risk aversion: experimental results," in Handbook of experimental economic results, ed. by C. Plott, and V. Smith, vol. 1, chap. 57. North Holland, Amsterdam.

Fox, C., And A. Tversky (1995): "Ambiguity aversion and comparative ignorance," Quarterly Journal of Economics, 110, 585-603. 
Gajdos, T., T. Hayashi, J.-M. Tallon, and J.-C. Vergnaud (2008): "Attitude toward imprecise information," Journal of Economic Theory, 140, 23-56.

Gilboa, I., And D. Schmeidler (1989): "Maxmin expected utility with a non-unique prior," Journal of Mathematical Economics, 18, 141-153.

Guiso, L., AND T. JAPPELLI (2008): "The role of intuition and reasoning in driving aversion to risk, aversion to ambiguity and regret," mimeo.

Guiso, L., And M. Paiella (2008): "Risk aversion, wealth, and background risk," Journal of the European Economic Association, 36, 1109-1150.

Harrison, G., M. Lau, and E. Rutström (2007): "Estimating risk attitudes in Denmark: a field experiment," Scandinavian Journal of Economics, 109, 341-368.

Harrison, G., M. LaU, and M. Williams (2002): "Estimating individual discount rates in Denmark: a field experiment," American Economic Review, 92, 1606-1617.

HAYASHI, T., AND R. WADA (forthcoming): "Choice with imprecise information: an experimental approach," Theory and Decision.

Hey, J., G. Lotito, and A. Maffioletti (2008): "The Descriptive and Predictive Adequacy of Theories of Decision Making Under Uncertainty/ Ambiguity," mimeo.

Ju, N., And J. MiaO (2008): "Ambiguity, learning, and asset returns," mimeo, Boston university.

Klibanoff, P., M. Marinacci, and S. Mukerji (2005): "A Smooth Model of Decision Making Under Uncertainy," Econometrica, (6), 1849-1892.

Lauriola, M., and I. Levin (2001): "Relating individual differences in attitude toward ambiguity to risky choices," Journal of Behavioral Decision Making, 14, 107-122.

Maccheroni, F., M. Marinacci, and A. Rustichini (2005): "Ambiguity aversion, Robustness, and the Variational Representation of Preferences," Econometrica, 74, 1447-1498.

Potamites, E., And B. Zhang (2007): "Measuring ambiguity attitudes: a field experiment among small-scale stock investors in China," mimeo, New York University.

Roux, N. (2008): "The Attitude Toward Probabilities of Portfolio Managers : an Experimental Study," Working paper 2008-73, Centre d'Economie de la Sorbonne - U. Paris I.

SchmeidleR, D. (1989): "Subjective probability and expected utility without additivity," Econometrica, 57(3), 571-587.

Tanaka, T., C. Camerer, and Q. Nguyen (2008): "Risk and time preferences: linking experimental and household survey data from Vietnam," Working paper.

VAN PraAg, B., AND A. BoolJ (forthcoming): "A simultaneous approach to the estimation of risk and time preferences," Journal of Economic Behavior and Organization.

von Gaudecker, H.-M., A. van Soest, and E. Wengstrom (2009): "Heterogeneity in risky choice behaviour in a broad population," Discussion paper 4022, IZA.

YATES, F., AND L. ZuKowski (1976): "Characterization of ambiguity in decision making," Behavioral Science, 21, 19-25. 